\title{
Long-term administration of nicorandil abolishes ischemic and pharmacologic preconditioning of the human myocardium: Role of mitochondrial adenosine triphosphate-dependent potassium channels
}

\author{
Mahmoud Loubani, FRCSI \\ Manuel Galiñanes, MD, PhD, FRCS
}

Background: Acute administration of mitochondrial adenosine triphosphate-dependent potassium channel openers preconditions the heart, but whether their long-term administration induces a permanent state of protection is unknown. These studies investigate the effect of long-term treatment with the mitochondrial adenosine triphosphate-dependent potassium channel opener nicorandil on the response of the human myocardium to ischemia and preconditioning.

Methods: Right atrial tissue obtained from patients regularly treated with or without nicorandil (mean of $20 \mathrm{mg} / \mathrm{d}$ for $18.6 \pm 2.5$ months) and undergoing cardiac surgery was sliced and equilibrated for 30 minutes and then subjected to 90 minutes of simulated ischemia, followed by 120 minutes of reoxygenation. In study 1 the following groups were studied to investigate the effect of nicorandil on the susceptibility of the myocardium to ischemia and on the protective effect of ischemic and pharmacologic preconditioning: (1) aerobic control; (2) simulated ischemia and reoxygenation alone; (3) ischemic preconditioning with 5 minutes of simulated ischemia and 5 minutes of reoxygenation; and (4) phenylephrine $(0.1 \mu \mathrm{mol} / \mathrm{L})$ for 5 minutes and 5 minutes' washout before simulated ischemia and reoxygenation. In study 2 the following groups were studied to investigate the effect of nicorandil on the responsiveness of mitochondrial adenosine triphosphate-dependent potassium channels: (1) aerobic control; (2) simulated ischemia and reoxygenation; (3) ischemic preconditioning; (4) diazoxide $(100 \mu \mathrm{mol} / \mathrm{L})$ for 10 minutes before simulated ischemia and reoxygenation, and (5) 5-hydroxydecanoate $(1 \mathrm{mmol} / \mathrm{L})$ for $10 \mathrm{~min}$ utes before simulated ischemia and reoxygenation. In study 3 the following groups were included to investigate the effect of the long-term administration of nicorandil on the kinase pathway involved in preconditioning: (1) aerobic control; (2) simulated ischemia and reoxygenation alone; (3) ischemic preconditioning; (4) phorbol 12 -myristate 13 -acetate $(1 \mu \mathrm{mol} / \mathrm{L})$, a protein kinase $\mathrm{C}$ activator, for 10 minutes before simulated ischemia and reoxygenation; and (5) anisomycin $(1 \mathrm{nmol} / \mathrm{L})$, a p38 mitogen-activated protein kinase activator, for 10 minutes before simulated ischemia and reoxygenation. At the end of each protocol, the leakage of creatine kinase (in units per gram wet weight) and the reduction of 3-[4,5 dimethylthiazol-2-yl]-2,5 diphenyltetrazolium bromide into insoluble formazan dye (in millimoles per gram wet weight) were measured.

Results: In study 1 the leakage of creatine kinase and the reduction of 3-[4,5 dimethylthiazol-2-yl]-2,5 diphenyltetrazolium bromide induced by simulated ischemia and reoxygenation were similar in the groups with or without nicorandil (creatine kinase, $3.4 \pm 0.1$ and $3.5 \pm 0.2$, respectively; 3-[4,5 dimethylthiazol-2yl]-2,5 diphenyltetrazolium bromide, $74.6 \pm 3.9$ and $67.9 \pm 7.3$, respectively; $P>$ .2 in each instance). Ischemic preconditioning and pharmacologic preconditioning protected the myocardium from patients without nicorandil (creatine kinase, $2.3 \pm$ 0.1 and $2.4 \pm 0.1$, respectively; 3-[4,5 dimethylthiazol-2-yl]-2,5 diphenyltetrazo- 
lium bromide, $131.4 \pm 4.9$ and $128.4 \pm 5.6$, respectively; $P<0.001$ vs simulated ischemia and reoxygenation alone in each instance) but not the myocardium from patients receiving nicorandil (creatine kinase, $3.3 \pm 0.1$ and $3.3 \pm 0.2$, respectively; 3-[4,5 dimethylthiazol-2-yl]-2,5 diphenyltetrazolium bromide, $89.7 \pm 6.5$ and $86.4 \pm 5.2$, respectively; $P>.2$ vs simulated ischemia and reoxygenation alone in each instance). In study 2 the administration of diazoxide had identical protection to that of ischemic preconditioning in the myocardium of patients not receiving nicorandil (creatine kinase, $2.1 \pm 0.2$ and $2.3 \pm 0.1$, respectively; 3-[4,5 dimethylthiazol-2-yl]-2,5 diphenyltetrazolium bromide, $141.4 \pm 7.4$ and $131.4 \pm 4.9$, respectively; $P<0.001$ vs simulated ischemia and reoxygenation alone in each instance) but failed to precondition the myocardium from patients treated with nicorandil (creatine kinase, $3.3 \pm 0.2$ and $3.4 \pm 0.1$, respectively; 3-[4,5 dimethylthiazol-2-yl]-2,5 diphenyltetrazolium bromide, $90.1 \pm 7.2$ and $86.4 \pm 5.2$, respectively; $P>0.2$ vs simulated ischemia and reoxygenation alone in each instance). In study 3 phorbol 12-myristate 13-acetate or anisomycin given for 10 minutes before simulated ischemia and reoxygenation afforded similar protection to that of ischemic preconditioning in the myocardium from patients with (creatine kinase, $1.5 \pm 0.3$ and $1.4 \pm 0.1$, respectively; 3-[4,5 dimethylthiazol-2-yl]-2,5 diphenyltetrazolium bromide, $147.0 \pm 4.9$ and $160.0 \pm 16.1$, respectively; $P<$ 0.001 vs simulated ischemia and reoxygenation alone in each instance) and without nicorandil (creatine kinase, $1.7 \pm 0.4$ and $1.4 \pm 0.2$, respectively; 3-[4,5 dimethylthiazol-2-yl]-2,5 diphenyltetrazolium bromide, $160.3 \pm 13.6$ and $158.3 \pm 11.8$, respectively; $P<.001$ vs simulated ischemia and reoxygenation alone in each instance).

Conclusion: The myocardium of patients chronically treated with nicorandil cannot be preconditioned either by ischemia or pharmacologically, and this is because of unresponsive mitochondrial adenosine triphosphate-dependent potassium channels. However, protection can be obtained by protein kinase $\mathrm{C}$ and $\mathrm{p} 38$ mitogen-activated protein kinase activation, which are downstream of mitochondrial adenosine triphosphate-dependent potassium channels in the signaling transduction pathway of preconditioning.

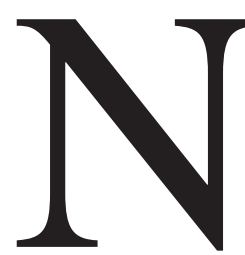

icorandil was first introduced in clinical practice in 1984 as the first in a new class of antianginal drugs. Since then, it has become widely used for the control of angina as part of combination therapy, and more recently, it is being increasingly used as the first-line and sole treatment in both stable ${ }^{1,2}$ and unstable angina. ${ }^{3}$ Nicorandil is a nicotinamide nitrate ester that has been shown to have an antianginal effect comparable with that of $\beta$-blockers and calcium antagonists. It has a bimodal mechanism of action combining 2 vasodilator mechanisms; it increases potassium conductance in the cell membrane, resulting in potassium outflow from the cell and causing membrane hyperpolarization ${ }^{4}$; and it also increases cellular levels of cyclic guanosine monophosphate, ${ }^{5}$ with both actions causing vasorelaxation. The nitrate-like action of nicorandil dilates epicardial coronary arteries, ${ }^{6}$ which results in an increase in the blood supply to the ischemic region of the myocardium. In addition, nicorandil has been shown to open adenosine triphosphate-dependent potassium $\left(\mathrm{K}_{\mathrm{ATP}}\right)$ channels in ischemic cardiomyocytes, ${ }^{7}$ and there is strong evidence that the mitochondrial, rather than the sarcolemmal, $\mathrm{K}_{\mathrm{ATP}}$ channels are involved in the protection of ischemic preconditioning, ${ }^{8-11}$ probably by decreasing the mitochondrial membrane potential. ${ }^{12,13}$ Yellon's laboratory has reported that nicorandil can mimic the protection of ischemic preconditioning, ${ }^{14}$ which has led to the suggestion that patients receiving nicorandil for the control of angina might be permanently protected. ${ }^{15}$

The aims of this study were to investigate the effects of long-term administration of nicorandil on the tolerance of the human myocardium to ischemia and on the protection of ischemic and pharmacologic preconditioning and on its signal transduction mechanism. 


\section{Methods}

\section{Patient Selection and Experimental Preparation}

Experiments were performed on muscle obtained from the right atrial appendage of patients undergoing elective coronary artery surgery in a cell necrosis model characterized in our laboratory and described previously. ${ }^{16}$ Patients were excluded if they had large atriums, atrial arrhythmias, poor left ventricular function (ejection fraction $<30 \%$ ), diabetes, and right ventricular failure or were receiving oral hypoglycemic agents, opioid analgesia, or catecholamines. Patients taking nicorandil had their last dose on the morning of the operation $2.5 \pm 0.4$ hours before harvesting of the atrial appendage. These patients were taking nicorandil for a mean of $18.6 \pm 2.5$ months at a mean dosage of $20 \mathrm{mg} / \mathrm{d}$. Local ethical committee approval was obtained for the harvesting technique. The specimens were collected in oxygenated N-2-hydroxyethylpiperazine-N-2-ethanesulfonic acid buffered solution at $4{ }^{\circ} \mathrm{C}$ to $5^{\circ} \mathrm{C}$ and immediately sectioned and prepared for study. The appendage was mounted onto a ground glass plate with the epicardial surface face down and then sliced freehand (to prevent crushing) with surgical skin graft blades (Shwann-Morton) to a thickness of between 300 and $500 \mu \mathrm{m}$. The muscles (weight, 30-50 mg) were then transferred to conical flasks (25-mL Erlenmeyer flasks, Schott Glaswerk) containing $10 \mathrm{~mL}$ of oxygenated buffered solution, and the flasks were placed in a shaking water bath maintained at $37^{\circ} \mathrm{C}$. The oxygenation of the incubation medium was maintained by a continuous flow of $95 \% \mathrm{O}_{2} / 5 \% \mathrm{CO}_{2}$ gas mixture to obtain a $\mathrm{PO}_{2}$ of between 25 and $30 \mathrm{kPa}$ and $\mathrm{PCO}_{2}$ of between 6 and $6.5 \mathrm{kPa}$. The $\mathrm{PO}_{2}, \mathrm{PCO}_{2}$, and $\mathrm{pH}$ in the incubation medium were monitored by means of intermittent analyses of the solution with an automated blood gas analyzer (model 855 Blood Gas System, Chiron Diagnostics), and the $\mathrm{pH}$ was kept at between 7.36 and 7.45. For the induction of simulated ischemia, the medium was bubbled with $95 \% \mathrm{~N}_{2} / 5 \% \mathrm{CO}_{2}$ (pH 6.80-7.00), and D-glucose was replaced with 2-deoxy glucose.

\section{Assessment of Tissue Injury and Viability}

Tissue injury was determined by measuring the leakage of creatine kinase (CK) into the incubation medium during the 120-minute reoxygenation period. This was assayed by using a kinetic UV method on the basis of the formation of NAD (Sigma Catalogue No. 1340-K), and the results were expressed as units per gram wet weight.

Tissue viability was assessed by the reduction of 3-[4,5 dimethylthiazol-2-yl]-2,5 diphenyltetrazolium bromide (MTT) to a blue formazan product at the end of the experimental time. The absorbance of the blue formazan formed was measured on a plate reader (Benchmark, Bio-Rad Laboratories) at $550 \mathrm{~nm}$, and the results were expressed as millimoles per gram wet weight. In this preparation the atrial tissue was not paced, and the force developed was not measured.

\section{Solutions and Drugs}

The incubation medium was prepared daily with deionized distilled water and contained the following: $\mathrm{NaCl}_{2}, 118 \mathrm{mmol} / \mathrm{L} ; \mathrm{KCl}$, $4.8 \mathrm{mmol} / \mathrm{L} ; \mathrm{NaHCO}_{3}, 27.2 \mathrm{mmol} / \mathrm{L} ; \mathrm{MgCl}_{2}, 1.2 \mathrm{mmol} / \mathrm{L}$; $\mathrm{KH}_{2} \mathrm{PO}_{4}, 1.0 \mathrm{mmol} / \mathrm{L} ; \mathrm{CaCl}_{2}, 1.25 \mathrm{mmol} / \mathrm{L} ;$ D-glucose, 10 $\mathrm{mmol} / \mathrm{L}$; and $\mathrm{N}$-2-hydroxyethylpiperazine-N-2-ethanesulfonic acid, $20 \mathrm{mmol} / \mathrm{L}$. During simulated ischemia, D-glucose was sub- stituted with 2-deoxy glucose (10 $\mathrm{mmol} / \mathrm{L})$ to maintain a constant osmolarity. Phenylephrine, prazosin, 5-hydroxydecanoate, and anisomycin were dissolved in deionized distilled water, whereas diazoxide and phorbol 12-myristate 13-acetate (PMA) were dissolved in dimethyl sulfoxide. Anisomycin is an antibiotic that inhibits protein synthesis and has been demonstrated to activate p38 mitogen-activated protein kinase (MAPK), whereas PMA is a phorbol ester that is widely used to activate protein kinase $\mathrm{C}$ (PKC). All the drug doses were chosen after extensive preliminary dose-response experiments. All reagents were obtained from Sigma.

\section{Experimental Protocols}

All atrial muscles were allowed to equilibrate under aerobic conditions for 30 minutes before 90 minutes of simulated ischemia and 120 minutes of reoxygenation. There were 6 specimens in each group from 6 different patients, with a total of 9 to 13 patients being enrolled in each study.

Study 1: To investigate the effect of long-term administration of nicorandil on myocardial tolerance to ischemia and on protection of ischemic and pharmacologic preconditioning with phenylephrine. Atrial muscles obtained from appendages of patients treated or not treated with nicorandil were randomly assigned to one of the following groups: (1) aerobic control; (3) simulated ischemia and reoxygenation alone; (3) ischemic preconditioning with 5 minutes of simulated ischemia and 5 minutes of reoxygenation; and (4) phenylephrine $(0.1 \mu \mathrm{mol} / \mathrm{L})$ for 5 minutes and 5 minutes' washout before simulated ischemia and reoxygenation.

Study 2: To investigate the effect of long-term administration of nicorandil on responsiveness of mitochondrial $K_{A T P}$ channels during preconditioning. Atrial slices obtained from appendages of patients treated or not treated with nicorandil were randomly assigned to one of the following groups: (1) aerobic control; (2) simulated ischemia and reoxygenation alone; (3) ischemic preconditioning with 5 minutes of simulated ischemia and 5 minutes of reoxygenation; (4) diazoxide $(100 \mu \mathrm{mol} / \mathrm{L})$, a mitochondrial $\mathrm{K}_{\mathrm{ATP}}$ channel opener, for 10 minutes before simulated ischemia and reoxygenation; and (5) 5-hydroxydecanoate $(1 \mathrm{mmol} / \mathrm{L})$, a mitochondrial $\mathrm{K}_{\mathrm{ATP}}$ channel blocker, for 10 minutes before simulated ischemia and reoxygenation.

Study 3: To investigate the effect of long-term administration of nicorandil on the kinase pathway involved in preconditioning. Atrial slices obtained from appendages of patients treated or not treated with nicorandil were randomly assigned to one of the following groups: (1) aerobic control; (2) simulated ischemia and reoxygenation alone; (3) ischemic preconditioning with 5 minutes of simulated ischemia and 5 minutes of reoxygenation; (4) PMA ( $1 \mu \mathrm{mol} / \mathrm{L})$, a PKC activator, for 10 minutes before simulated ischemia and reoxygenation; and (5) 
anisomycin ( $1 \mathrm{nmol} / \mathrm{L})$, a p38MAPK activator, for 10 minutes before simulated ischemia and reoxygenation.

\section{Statistical Analysis}

All data are presented as means \pm SEM. Mean values were compared by means of analysis of variance with application of the post hoc Tukey test.

\section{Results}

Effect of Long-Term Administration of Nicorandil on Myocardial Tolerance to Ischemia and on Protection of Ischemic and Pharmacologic Preconditioning with Phenylephrine (Study 1)

As shown in Figure 1, the increased CK leakage and decreased MTT reduction induced by ischemia and reoxygenation were similar in the muscles from patients with and without long-term nicorandil treatment. Figure 1 also shows that ischemic and pharmacologic preconditioning with phenylephrine resulted in identical protection in the nicorandil-free group but failed to protect the myocardium in the nicorandil-treated group.

\section{Effect of Long-Term Administration of Nicorandil on Responsiveness of Mitochondrial $\mathrm{K}_{\mathrm{ATP}}$ Channels During Preconditioning (Study 2)}

As expected, Figure 2 shows that the selective opening of mitochondrial $\mathrm{K}_{\mathrm{ATP}}$ channels with diazoxide resulted in protection (ie, reduced CK leakage and increased MTT reduction) similar to that provided by ischemic preconditioning, with no detrimental effect beyond that of ischemia and reoxygenation alone when the channels were blocked with 5-hydroxydecanoate. In contrast with these results, diazoxide did not protect the myocardium in the nicorandiltreated group.

\section{Effect of Long-Term Administration of Nicorandil on the Kinase Pathway Involved in Preconditioning (Study 3)}

The results shown in Figure 3 demonstrate that activation of PKC and p38MAPK resulted in similar protection as that provided by ischemic preconditioning, as shown on the basis of the CK leakage and MTT reduction values in both the nicorandil-free and nicorandil-treated groups.

\section{Discussion}

The present studies have demonstrated that the long-term administration of nicorandil, a mitochondrial $\mathrm{K}_{\mathrm{ATP}}$ channel opener ${ }^{14}$ and nitric oxide donor, ${ }^{17}$ abolishes the ability of the human myocardium to be protected by ischemic and pharmacologic preconditioning without exacerbating the susceptibility to ischemic injury. In addition, they have shown that the likely cause of the failure to precondition the myocardium of patients receiving nicorandil is the unre- sponsiveness of the mitochondrial $\mathrm{K}_{\mathrm{ATP}}$ channels because protection cannot be obtained with diazoxide, a specific mitochondrial $\mathrm{K}_{\mathrm{ATP}}$ channel opener, but can be elicited by activation of PKC and p38MAPK, which are downstream of mitochondrial $\mathrm{K}_{\mathrm{ATP}}$ channels in the signaling transduction cascade of preconditioning. ${ }^{18}$ These results have important clinical implications and shed light onto the mechanism of protection by preconditioning that are discussed below.

Opening of the mitochondrial $\mathrm{K}_{\mathrm{ATP}}$ channels has been demonstrated to be an obligatory step in the signal transduction mechanism of preconditioning. 8,9,11 Thus nicorandil and other mitochondrial $\mathrm{K}_{\mathrm{ATP}}$ channel openers have been shown to mimic the cardioprotection of ischemic preconditioning when given acutely (ie, immediately before the ischemic insult) in both animal ${ }^{7,11,15}$ and human ${ }^{8,9}$ studies. As a result, and as suggested by Carr and Yellon, ${ }^{15}$ one might be tempted to hypothesize that the long-term administration of mitochonrial $\mathrm{K}_{\mathrm{ATP}}$ channel openers induces a permanent state of protection against ischemic injury. However, the present studies are the first to report that when mitochonrial $\mathrm{K}_{\mathrm{ATP}}$ channel openers are given on a longterm basis, as occurs clinically, these channels become unresponsive, with loss of the cardioprotection of preconditioning.

Our findings contrast with those reported by Carr and Yellon ${ }^{15}$ showing that the long-term administration of nicorandil is actually protective. Their and our studies used human atrial myocardium, and the only difference between the 2 studies is that they assessed recovery of contractile function in their study as opposed to tissue viability in ours, which might offer an explanation as to the differing results. Further fuel is added to the controversy when the same authors observed that the protection of the myocardium of patients receiving long-term nicorandil is in fact abolished by the application of ischemic preconditioning. These results require clarification because it is difficult to understand how 2 protective interventions using identical signal transduction mechanisms can annul each other.

The mechanism by which the long-term administration of nicorandil renders the mitochonrial $\mathrm{K}_{\mathrm{ATP}}$ channels unresponsive to preconditioning with ischemia and with diazoxide is not completely elucidated by the present studies, but they have shown that the activation of kinases that we have previously shown to be downstream of mitochonrial $\mathrm{K}_{\mathrm{ATP}}$ channels $^{18}$ is unaffected because their activation can still elicit protection. It has been suggested that the generation of free radical species is the link between mitochonrial $\mathrm{K}_{\mathrm{ATP}}$ channels and the activation of PKC. ${ }^{11}$ If this is the case, then it might be speculated that a permanent opening state of mitochonrial $\mathrm{K}_{\mathrm{ATP}}$ channels results in a reduction in the formation of free radicals, a thesis that gains support from the demonstration that nicorandil possesses antioxidant properties. ${ }^{19}$ 

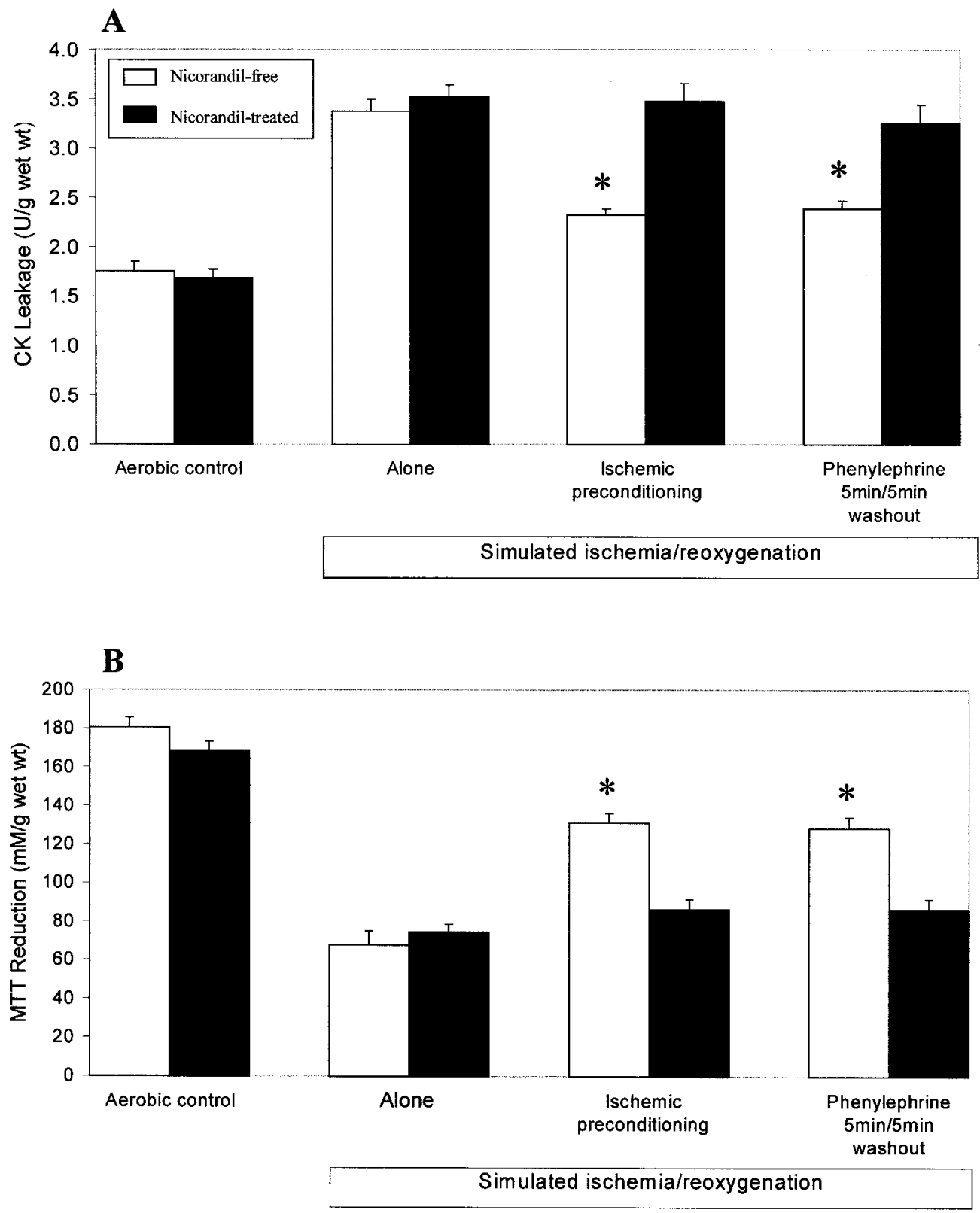

Figure 1. CK leakage into the media: $A$, during the 120 -minute reoxygenation period and MTT reduction by the slices; $B$, at the end of the reoxygenation period in human atrial myocardium subjected to various protocols (see text for details) to investigate the effect of the long-term administration of nicorandil on the myocardial tolerance to ischemia and on the protection of ischemic and pharmacological preconditioning with phenylephrine (study 1). Data are expressed as means \pm SEM of 6 experiments. ${ }^{*} P<.001$ versus simulated ischemia and reoxygenation alone.

The present studies raise fundamental questions on the clinical utility and safety of nicorandil and other mitochonrial $\mathrm{K}_{\mathrm{ATP}}$ channel openers for the control of angina symptoms. The permanent opening of the mitochonrial $\mathrm{K}_{\mathrm{ATP}}$ channels deprive the heart of the intrinsic protective mechanism of preconditioning that might be a risk factor in the presence of ischemic heart disease. Therefore if the bene- ficial action of nicorandil is solely due to its nitrate effect, then the use of this compound might not be fully justified. However, if, as discussed earlier, the maintenance of mitochonrial $\mathrm{K}_{\mathrm{ATP}}$ channels in an open state might reduce the generation of free radicals and oxidative stress, then these beneficial effects of nicorandil might counterbalance the loss of preconditioning. Indeed, oxidative stress has been 


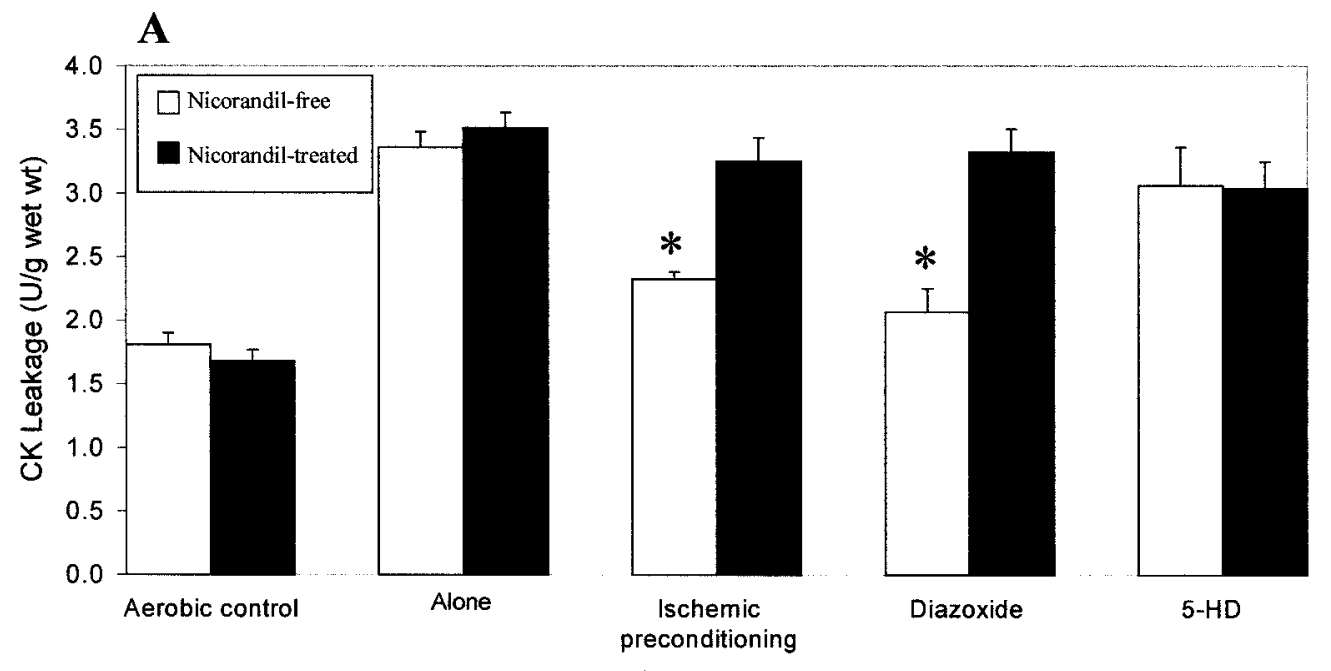

Simulated ischemia/reoxygenation

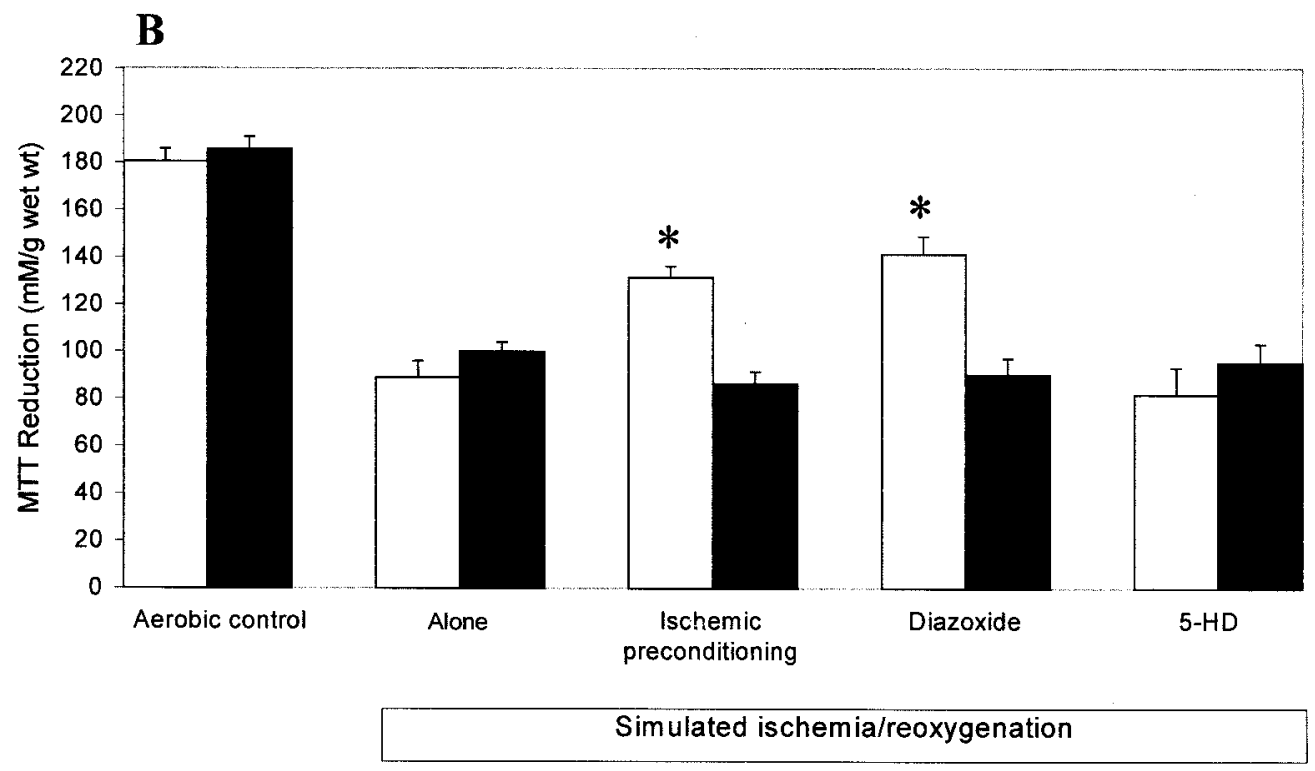

Figure 2. CK leakage into the media: $A$, during the 120 -minute reoxygenation period and MTT reduction by the slices; $B$, at the end of the reoxygenation period in human atrial myocardium subjected to various protocols (see text for details) to investigate the effect of the long-term administration of nicorandil on the responsiveness of mitochondrial $K_{\text {ATP }}$ channels during preconditioning (study 2). Data are expressed as means \pm SEM of 6 experiments. ${ }^{*} P<.001$ versus simulated ischemia and reoxygenation alone. 5-HD, 5-Hydroxydecanoate.

suggested as an important mechanism of many disease states, including the inflammatory response in diabetes, ${ }^{20}$ atherosclerosis, ${ }^{21}$ cardiac hypertrophy, ${ }^{22}$ and heart failure. ${ }^{23}$ It is clear that further experimental and clinical studies are required to fully elucidate the mechanism and the clinical repercussions of nicorandil and similar agents on ischemic heart disease and ischemic syndromes.
It is necessary to mention that our studies were performed in an in vitro preparation that was not electrically stimulated (ie, nonbeating), and it was not possible to obtain functional data. Therefore any extrapolation to the clinical setting should be made with caution. Another potential limitation of our studies was the use of atrial, as opposed to ventricular, myocardial tissue, and again, any extrapolation 

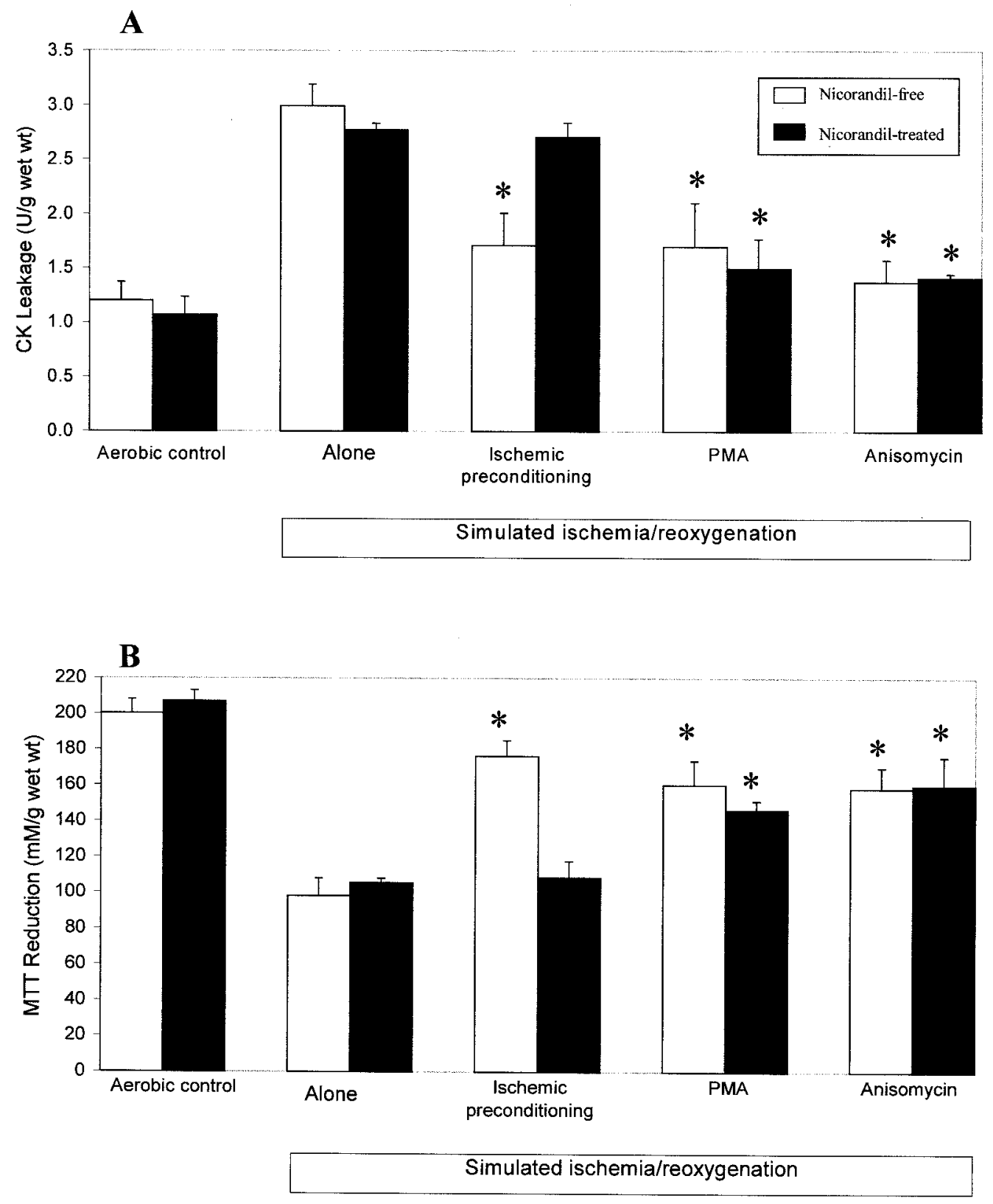

Figure 3. CK leakage into the media: $A$, during the 120-minute reoxygenation period and MTT reduction by the slices; $B$, at the end of the reoxygenation period in human atrial myocardium subjected to various protocols (see text for details) to investigate the effect of the long-term administration of nicorandil on the kinase pathway involved in preconditioning (study 3). Data are expressed as means \pm SEM of 6 experiments. ${ }^{*} P<.001$ versus simulated ischemia and reoxygenation alone.

of the conclusions from these studies to the ventricular myocardium should also be made with caution.

Although these studies have demonstrated the unresponsiveness of the mitochonrial $\mathrm{K}_{\mathrm{ATP}}$ channels as the cause of failure to precondition the myocardium of nicorandil-treated patients, they show that protection can still be obtained by direct activation of PKC or p38MAPK, which are down- stream of mitochonrial $\mathrm{K}_{\mathrm{ATP}}$ channels in the signal transduction pathway of ischemic preconditioning. Therefore protection can be elicited by bypassing the steps of the transduction cascade that might be affected by disease states, such as diabetes or heart failure, ${ }^{24}$ or by medication, such as sulphonylureas, ${ }^{24}$ and antianginal treatment, such as nicorandil, as shown from the current studies. Undoubtedly 
greater understanding of the various elements participating in the signal transduction pathway and identification of agents with selective activity on factors to avoid unwanted side effects will be required before its use might be considered in the clinical setting.

\section{References}

1. Krumenacker M, Roland EO. Clinical profile of nicorandil: an overview of its haemodynamic properties and therapeutic efficacy. J Cardiovasc Pharmacol. 1992;20:S93-102.

2. Frampton J, Buckley M, Fitton A. Nicorandil: a review of its pharmacology and therapeutic efficacy in angina pectoris. Drugs. 1992; 44:625-55.

3. Patel DJ, Purcell HJ, Fox KM. Cardioprotection by opening of the $\mathrm{K}_{\text {ATP }}$ channel in unstable angina. Eur Heart J. 1999;20:51-7.

4. Cook NS. The pharmacology of potassium channels and their therapeutic potential. Trends Pharmacol Sci. 1988;9:21-8.

5. Flaherty JT. Nitrate tolerance: a review of the evidence. Drugs. 1989;37:523-50.

6. Suryapranata H, MacLeod D. Nicorandil and cardiovascular performance in patients with coronary artery disease. $J$ Cardiovasc Pharmacol. 1992;20:S45-51.

7. Hiraoka M, Fan Z. Activation of ATP-sensitive outward potassium current by nicorandil (2-nicotinamidoethyl-nitrate) in isolated ventricular myocytes. J Pharmacol Exp Ther. 1989;250:278-85.

8. Speechly-Dick M, Grover G, Yellon DM. Does ischaemic preconditioning in the human involve protein kinase $\mathrm{C}$ and the ATP-dependent $\mathrm{K}^{+}$channel? Circ Res. 1995;77:1030-5.

9. Ghosh S, Standen N, Galiñanes. Evidence for mitochondrial channels as effectors of human myocardial preconditioning. Cardiovasc Res. 2000;45:934-40.

10. Ghosh S, Standen N, Galiñanes M. Preconditioning the human myocardium by simulated ischemia: studies on the early and delayed protection. Cardiovasc Res. 2000;45:339-50.

11. Pain T, Yang X, Critz SD, et al. Opening of mitochondrial KATP channels triggers the preconditioned state by generating free radicals. Circ Res. 2000;87:460-6.

12. Holmuhamedov EL, Jovanovic S, Dzeja PP, Jovanovic A, Terzic A. Mitochondrial ATP-sensitive $\mathrm{K}+$ channels modulate cardiac mitochondrial function. Am J Physiol Heart Circ Physiol. 1998;275: H1567-76.
13. Liu Y, Sato T, Seharaseyon J, Szewczyk A, O’Rourke B, Marban E. Mitochondrial ATP-dependent potassium channels. Viable candidate effectors of ischemic preconditioning. Ann N Y Acad Sci. 1999;874: 27-37.

14. Imagawa J, Baxter GF, Yellon DM. Myocardial protection afforded by nicorandil and ischaemic preconditioning in a rabbit infarct model in vivo. J Cardiovasc Pharmacol. 1998;31:74-9.

15. Carr CS, Yellon DM. Ischaemic preconditioning may abolish the protection afforded by ATP-sensitive potassium channel openers in isolated human atrial muscle. Basic Res Cardiol. 1997;92:25-260.

16. Zhang JG, Ghosh S, Ockelford C, Galiñanes M. Characterization of an in vitro model for the study of the short and prolonged effects of myocardial ischaemia and reperfusion in man. Clin Sci. 2000;99:33950.

17. Nishikawa Y, Kanki H, Ogawa S. Differential effects of N-acetylcysteine on nitroglycerin- and nicorandil-induced vasodilatation in human coronary circulation. J Cardiovasc Pharmacol. 1998;32:21-8.

18. Loubani M, Galiñanes M. MitoK ${ }_{\mathrm{ATP}}$ channels are upstream of PKC and $\mathrm{p} 38 \mathrm{MAPK}$ activation in the preconditioning of the human myocardium by alpha 1 adrenoceptors [abstract]. Eur Heart J. 2001;22: 384.

19. Naito A, Aniya Y, Sakanashi M. Antioxidative action of nitrovasodilator nicorandil: inhibition of oxidative activation of liver microsomal glutathione S-transferase and lipid peroxidation. Jpn J Pharmacol. 1994;65:209-13.

20. Matata BM, Galiñanes M. Cardiopulmonary bypass exacerbates oxidative stress but does not increase proinflammatory cytokine release in patients with diabetes compared with patients without diabetes: regulatory effects of exogenous nitric oxide. J Thorac Cardiovasc Surg. 2000;120:1-11.

21. Langlois M, Duprez D, Delanghe J, De Buyzere M, Clement DL. Serum vitamin $C$ concentration is low in peripheral arterial disease and is associated with inflammation and severity of atherosclerosis. Circulation. 2001;103:1863-8.

22. Nagueh SF, Stetson SJ, Lakkis NM, et al. Decreased expression of tumor necrosis factor-alpha and regression of hypertrophy after nonsurgical septal reduction therapy for patients with hypertrophic obstructive cardiomyopathy. Circulation. 2001;103:1844-50.

23. Ferdinandy P, Danial H, Ambrus I, Rothery RA, Schulz R. Peroxynitrite is a major contributor to cytokine-induced myocardial contractile failure. Circ Res. 2000;87:241-7.

24. Ghosh S, Standen NB, Galiñanes M. Failure to precondition pathological human myocardium. J Am Coll Cardiol. 2001;37:711-8. 